\title{
Delayed surgery after histologic or radiologic-diagnosed clinical stage I lung adenocarcinoma
}

\author{
Chien-Sheng Huang ${ }^{1,2}$, Po-Kuei Hsu ${ }^{1}$, Chun-Ku Chen ${ }^{3}$, Yi-Chen Yeh ${ }^{4}$, Chun-Che Shih ${ }^{2,5}$, Biing-Shiun \\ Huang ${ }^{1}$
}

${ }^{1}$ Division of Thoracic Surgery, Department of Surgery, Taipei Veterans General Hospital, Taipei; ${ }^{2}$ Institute of Clinical Medicine, School of Medicine, National Yang-Ming University, Taipei; ${ }^{3}$ Department of Radiology, ${ }^{4}$ Department of Pathology, ${ }^{5}$ Division of Cardiovascular Surgery, Department of Surgery, Taipei Veterans General Hospital, Taipei

Contributions: (I) Conception and design: CS Huang; (II) Administrative support: CC Shih, BS Huang; (III) Provision of study materials or patients: CS Huang, CK Chen, YC Yeh; (IV) Collection and assembly of data: CS Huang, PK Hsu; (V) Data analysis and interpretation: CS Huang, PK Hsu; (VI) Manuscript writing: All authors; (VII) Final approval of manuscript: All authors.

Correspondence to: Chien-Sheng Huang, MD. Division of Thoracic Surgery, Department of Surgery, Taipei Veterans General Hospital, and Institute of Clinical Medicine, School of Medicine, National Yang-Ming University, 201, Section 2, Shih-Pai Road, Taipei. Email: huangcs@vghtpe.gov.tw.

Background: The impact of delayed surgery on clinical outcomes after histologic or radiologic diagnosis of clinical stage I adenocarcinoma remains controversial. We evaluated the effects of delayed surgery on outcomes of patients with early-stage lung cancer.

Methods: Associations between time intervals of "histologic diagnosis-to-surgery" (HDS), "radiologic diagnosis-to-surgery" (RDS), and overall survival in clinical stage I adenocarcinoma were assessed using multivariable Cox proportional hazard analysis.

Results: A total of 561 consecutive patients with preoperative histologic confirmation of stage I lung cancer between 2006 and 2016 were included. Median time to HDS and RDS were 20 (2-267) and 58 (38-2,983) days. Higher Charlson comorbidity score, receiving brain magnetic resonance imaging screening, and video-assisted thoracoscopic surgery approach were significantly associated with increased risk of late HDS (>21 days). Smaller tumor size and non-radiologic solid-dominant pattern were significantly associated with increased risk of late RDS (>60 days). In the overall cohort, worse 5-year overall survival was associated with late HDS compared to early HDS (75.9\% vs. $85.5 \%, \mathrm{P}=0.003)$. No significant differences were found in later late $v s$. early $\mathrm{RDS}(83.7 \%$ vs. $83.3 \%, \mathrm{P}=0.570)$. In 286 propensity-score matched patients, late HDS [adjusted hazard ratio $(\mathrm{aHR})=2.031, \mathrm{P}=0.038$ ], higher Charlson comorbidity score $(\mathrm{aHR}=1.610, \mathrm{P}=0.023)$, larger tumor size $(\mathrm{aHR}=2.164, \mathrm{P}=0.031)$, without brain magnetic resonance imaging screening $(\mathrm{aHR}=2.051$, $\mathrm{P}=0.045)$, and tumor with angiolymphatic invasion $(\mathrm{aHR}=4.638, \mathrm{P}=0.001)$ were significantly associated with lower overall survival.

Conclusions: In patients with stage I lung adenocarcinoma, delayed surgery after a histologic diagnosis is an independent predictor of overall survival after adjusting for clinical risk factors, suggesting meaningful differences in clinical outcomes between timely $v s$. delayed surgeries.

Keywords: Non-small cell lung cancer; histologic diagnosis; radiologic diagnosis; delayed surgery

Submitted Aug 15, 2019. Accepted for publication Dec 26, 2019.

doi: $10.21037 /$ jtd.2019.12.123

View this article at: http://dx.doi.org/10.21037/jtd.2019.12.123

(C) Journal of Thoracic Disease. All rights reserved. 


\section{Introduction}

The timeliness of diagnosis and treatment is increasingly recognized as an important factor in quality of care, while extended waiting times and delays in appropriate care may negatively affect morbidity, mortality, and quality of life (1). Lung cancer remains the leading cause of cancerrelated deaths worldwide (2), and many patients in different facilities and countries experience substantial waits for lung cancer diagnosis and subsequent treatment.

Currently, surgical resection remains the gold-standard of treatment in early-stage lung cancer. Unsurprisingly, patients who are diagnosed as both early-stage lung cancers and surgical candidates usually require more accurate diagnostic and preoperative testing before treatment initiation than advanced lung cancer patients who will not undergo surgery $(3,4)$. This is because the results of diagnostic testing must be conclusive in order to support appropriate treatment decisions. For instance, invasive mediastinal lymph node evaluation, such as mediastinoscopy or endobronchial ultrasonography (EBUS)-guided lymph node aspiration will often be performed to confirm or rule out suspected mediastinal lymph node metastasis after a preoperative work-up has been done, including staging. Time-consuming tests, including positron emission tomography/computed tomography (PET/CT) and brain magnetic resonance imaging (MRI) screening $(5,6)$, may result in diagnosis-to-treatment delays that may, in turn, delay the impending surgery.

The consequence of delayed surgery on patient outcomes, especially in early-stage lung cancer patients, is controversial. Some studies have suggested that such delays will have no significant impact on overall survival (OS) $(7,8)$, while other studies have demonstrated associations between shorter waiting times and increased survival (9-11). The impact of delayed surgery on outcomes is difficult to interpret, and may possibly be due to ambiguous definitions of intervals between lung cancer diagnoses and the timing of subsequent surgeries-that is the "diagnosis-to-surgery" interval. For example, different studies have reported different methods of confirming an initial diagnosis, including radiologic $(8,12)$; histologic $(7,9)$, preoperative or intraoperative histologic diagnoses (3); mixed radiologic and histologic confirmation $(11,13-16)$ or other diagnostic testing $(5,10,17,18)$; which may delay surgery to different degrees.

Therefore, the present study aimed to evaluate the impact of delayed surgery on patient outcomes after histologic or radiologic diagnosis of clinical early-stage lung cancer.

\section{Methods}

\section{Patient selection}

The medical records of patients who underwent pulmonary resection for clinical stage I non-small cell lung carcinoma (NSCLC) from January 2006 to December 2016 at Taipei Veterans General Hospital were reviewed. Clinical and demographic characteristics, including age, sex, smoking history, pulmonary function, preoperative serum carcinoembryonic antigen (CEA) level, date of histologic diagnosis, date of radiological diagnosis, histologic tumor type, tumor size, presence of lymphovascular invasion, presence of pleural invasion, and surgical approach were recorded. The study protocol was approved by the hospital Institutional Review Board and the informed consent requirement was waived (approval no. 2019-04-009AC). During the study period, 1,428 patients who underwent pulmonary resection for clinical stage I NSCLC. Exclusion criteria included: non-adenocarcinoma patients $(n=122)$ and patients who had both surgery and histologic diagnosis (intraoperative) on the same day $(n=745)$. Finally, data of 561 patients were included for further analysis.

\section{Definitions of terms used in the study}

"Date of histologic diagnosis" was defined as the date of the procedure performed (CT-guided or transbronchial biopsy) for NSCLC histologic confirmation. "Date of radiologic diagnosis" was the date of the first chest CT when the initial lung tumor could be recognized in our imaging system. "Histologic diagnosis-to-surgery" (HDS) and radiologic diagnosis-to-surgery" (RDS) intervals were defined as time (days) between the histologic or radiologic diagnosis date and operative date, respectively. Limited pulmonary function was defined as preoperative predicted forced expiratory volume in one second (FEV1) or diffusion lung capacity for carbon monoxide (DLCO); either one $<50 \%$.

\section{Early vs. late surgical treatment}

For each patient, the numbers of "HDS" and "RDS" interval days, categorized as "early" (1-21 days) or "late" HDS (>21 days) intervention, which were based on a median time to surgery of 20 days at an initial evaluation of the timing of surgery distribution in the overall cohort, were identified. The cut-off point was 21 days, given the completion time for diagnostic work-up and staging. 
Different cutoff points, such as $2-5$ weeks, were also used in analysis of the overall cohort. "Early" or "late" RDS was based on a similar definition. The median time from the first chest CT to operation was 58 days and the RDS cutoff was defined as 60 days.

\section{Preoperative radiological evaluation}

Radiologic tumor findings were defined by thin-section CT or the multidimensional slicing and reconstruction into axial, coronal, and sagittal views. Tumor characteristics from the latest preoperative chest CT were read by two independent observers and the tumor size was reviewed in detail. Based on the consolidation tumor ratio (CTR), partsolid tumor was defined as one with both a focal nodular opacity and GGO (0 less than CTR $\leq 1.0)$, classed into two groups: GGO-dominant (0 less than CTR 0.5) and soliddominant $(0.5$ less than CTR $\leq 1.0)(19)$.

\section{Follow up}

Operative mortality included death from all causes occurring during surgery or within 30 days after surgery but during the same hospitalization period. Postoperative surveillance was scheduled every 3 months for the first 2 years, every 6 months for the third to fifth year, and annually thereafter. Chest CT was performed every 6 months for 5 years, then annually. The primary outcome was OS. In HDS analysis, OS was defined as the interval between the date of tissue diagnosis and the date or either death or last follow-up (OS-HDS). In RDS analysis, OS was defined as the interval between the date of first chest CT image and the date or either death or last follow-up (OSRDS). An observation was censored at the last follow-up session when the patient had either died without recurrence or was lost-to-follow-up. As of January 31, 2019, all patients had been followed-up except for 36 patients lost to followup (follow-up rate 93.6\%; 18 patients each in early and late HDS groups).

\section{Statistical analysis}

All continuous data are expressed as means and standard deviations (SD). Categorical variables were analyzed by Chisquare test or Fisher's exact test and continuous variables by two-sample $t$ test. Survival curves were calculated by the Kaplan-Meier method. To reduce selection bias and make the two groups more similar, a 1:1 propensity score matching (PSM) was conducted using different baseline characteristics between groups, including age, comorbidity score, limited pulmonary function, preoperative brain MRI screening and VATS approach. Cox regression analysis was then performed on the matched sample for predictors of OS. Predictors with $\mathrm{P}$ values $\leq 0.1$ in univariate analyses were included in the multivariate model. Statistical analyses were performed using SPSS Statistics for Windows, Version 22.0 (IBM Corp., Armonk, NY).

\section{Results}

The median follow-up period after histologic and radiologic diagnoses were 56.2 and 60.1 months, respectively, during which the following occurred: 30 -day postoperative death in $1(0.18 \%)$ patient, death from diseases other than lung cancer in 30 (5.3\%), and tumor recurrence in 103 (18.4\%). Locoregional-only recurrences, distal recurrences, and distant with local recurrences occurred in 46 (44.6\%), 25 (24.2\%), and $32(31.1 \%)$ patients, respectively. Median HDS interval was 20 days [interquartile range (IQR): 13-29 days] and for RDS was 58 days (IQR: 38-113.5 days).

As shown in Table 1, overall, 254 males (45.3\%) and 307 females $(54.7 \%)$ were included. Mean patient age was $63.4(\mathrm{SD}=11.1)$ years. The majority of patients were asymptomatic, had preoperative whole-body PET/CT scan to rule out mediastinal and distant metastasis, received preoperative brain MRI screening, underwent lobectomy (or above) surgical intervention, and underwent pulmonary resection by VATS approach. The majority of tumors were pathologically diagnosed as invasive adenocarcinomas and most were at pathological TNM stage pT2a (IB, 300, $53.5 \%)$. A total of 49 patients (8.7\%) had stage migration determined pathologically (20 upstaged to stage IIB, 14 to stage IIIA, 1 to stage IIIB and 14 to stage IV). A total of 321 patients $(57.2 \%)$ and 240 patients $(42.8 \%)$ were in early and late HDS groups after histologic diagnosis, respectively. The early and late RDS groups after histologic diagnosis included $292(52.0 \%)$ and $269(48.0 \%)$ patients, respectively. Age, Charlson comorbidity score, limited pulmonary function, MRI screening, and VATS approach were significantly different between the early and late HDS groups $(\mathrm{P}<0.05)$. Age, Charlson comorbidity score, tumor size, surgical approach, solid-dominant pattern, and VATS approach were significantly different between the early and late RDS groups.

Table 2 shows the independent predictors of late HDS overall. The univariate model revealed that age, Charlson 
Table 1 Demographics of patients with clinical stage I adenocarcinoma undergoing either early or delayed pulmonary resection after histologic or radiologic diagnoses

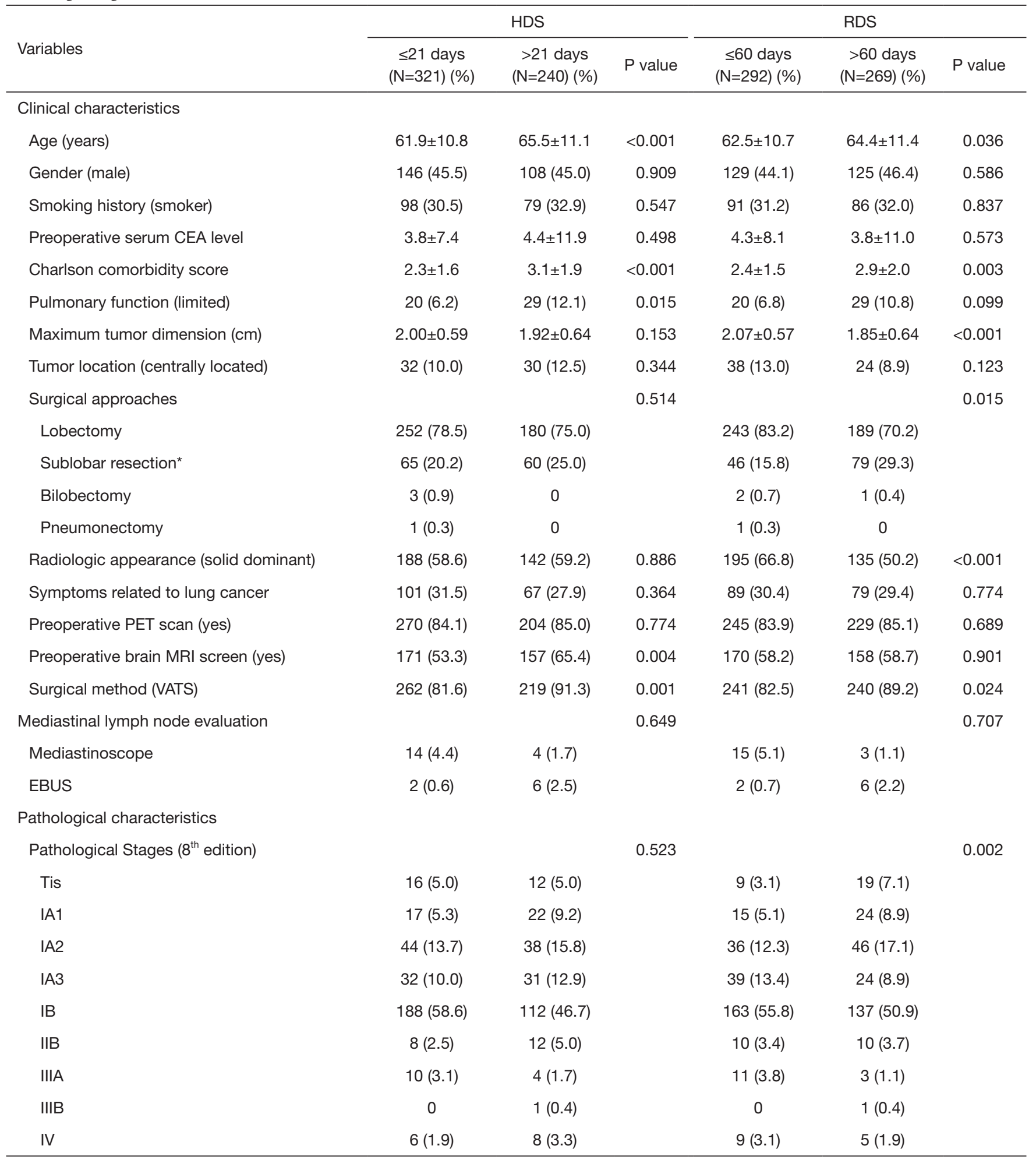

HDS, histologic diagnosis to surgery; RDS, radiologic diagnosis to surgery; CEA, carcinoembryonic antigen; *Sublobar resection, wedge + segmentectomy; PET, positron emission tomography; MRI, magnetic resonance imaging; VATS, video-assisted thoracoscopic surgery; EBUS, endobronchial ultrasound. 
Table 2 Independent predictors of late HDS in 561 clinical stage I adenocarcinoma patients

\begin{tabular}{|c|c|c|c|c|c|c|}
\hline Variables & \multicolumn{3}{|c|}{ Univariate } & \multicolumn{3}{|c|}{ Multivariate } \\
\hline Age (years) & 1.030 & $1.014-1.047$ & $<0.001$ & 0.996 & $0.971-1.022$ & 0.763 \\
\hline Gender (male) & 0.981 & $0.701-1.372$ & 0.909 & - & - & - \\
\hline Smoking history (smoker) & 1.117 & $0.780-1.599$ & 0.547 & - & - & - \\
\hline Charlson comorbidity score & 1.282 & $1.159-1.419$ & $<0.001$ & 1.299 & $1.103-1.530$ & 0.002 \\
\hline Pulmonary function (limited) & 2.068 & $1.139-3.755$ & 0.017 & 1.498 & $0.794-2.826$ & 0.212 \\
\hline Maximum tumor dimension (cm) & 0.819 & $0.623-1.077$ & 0.153 & - & - & - \\
\hline Tumor location (central located) & 1.290 & $0.760-2.189$ & 0.345 & - & - & - \\
\hline Preoperative PET/CT (with) & 1.070 & $0.673-1.702$ & 0.774 & - & - & - \\
\hline Recipient of brain MRI screening & 1.659 & $1.175-2.342$ & 0.004 & 1.570 & $11.075-2.292$ & 0.019 \\
\hline EBUS lymph node aspiration & 4.090 & $0.818-20.443$ & 0.086 & 3.017 & $0.586-15.534$ & 0.187 \\
\hline Surgical method (VATS) & 2.348 & $1.383-3.987$ & 0.002 & 1.967 & $1.112-3.480$ & 0.020 \\
\hline Wedge resection (Yes) & 1.663 & $1.053-2.627$ & 0.029 & 1.414 & $0.876-2.282$ & 0.156 \\
\hline
\end{tabular}

${ }^{1}$ Calculated by Logistic regression method; only variables with $\mathrm{P} \leq 0.1$ after the univariate analyses were entered into the multivariate model. HDS, histologic diagnosis to surgery; OR, odds ratio; $\mathrm{Cl}$, confidence interval; aOR, adjusted odds ratio.

comorbidity score, limited pulmonary function, receiving brain MRI screening, VATS approach, and wedge resection were significantly associated with increased risk of delayed HDS. In multivariate analysis, higher Charlson comorbidity score [adjusted odds ratio $(\mathrm{aOR})=1.299, \mathrm{P}=0.002$ ], recipient of brain MRI screening ( $\mathrm{aOR}=1.570, \mathrm{P}=0.019)$, and undergoing the VATS approach $(\mathrm{aOR}=1.967, \mathrm{P}=0.020)$ were significantly associated with increased risk of late HDS. Multivariate analysis revealed that smaller tumor size and non-radiologic solid-dominant pattern were independent predictors of increased risk of late RDS (Table S1).

Figure 1 demonstrates the OS of the histologic (A, OSHDS) and radiologic (B, OS-RDS) diagnoses between early and late interventions. Among the histologic confirmation groups, 5 -year OS-HDS for early and late HDS was $85.5 \%$ vs. $75.9 \%(\mathrm{P}=0.003)$. Among the radiologic diagnosis group, 5 -year OS-RDS for early and late RDS was $83.3 \%$ vs. $83.7 \%(\mathrm{P}=0.570)$.

Table 3 demonstrates the PSM between earlier and late HDS. The matched sample included 143 patients who each underwent surgery with early- and late-HDS, respectively. No statistically significant differences were found in any clinical variable between groups after matching except fewer lymph node dissections in the late-HDS group. OS risk analyses in the PSM cohort are presented in Table 4. Univariate models revealed that lateHDS ( $\mathrm{HR}=2.276, \mathrm{P}=0.011$ ), age, preoperative CEA level, Charlson comorbidity score, tumor size, central location of tumor, radiologic appearance with solid-dominant pattern, symptoms related to lung cancer, without preoperative brain MRI screening, TNM pathological stage, tumor presentation of angiolymphatic invasion, high grade predominant adenocarcinoma and receiving adjuvant chemotherapy were significantly associated with higher OS. After adjusting for confounders in the multivariate model, late-HDS ( $>21$ days) remained significantly associated with lower OS (aHR=2.031, 95\% CI: 1.041-3.963, $\mathrm{P}=0.038$ ). Similar results were also observed using a higher late HDS cutoff ( $>28$ days; aHR=1.845, $\mathrm{P}=0.048$ ). OS after PSM is shown in Figure 2.

\section{Discussion}

Waiting times for treatment in cancer care continue to be 

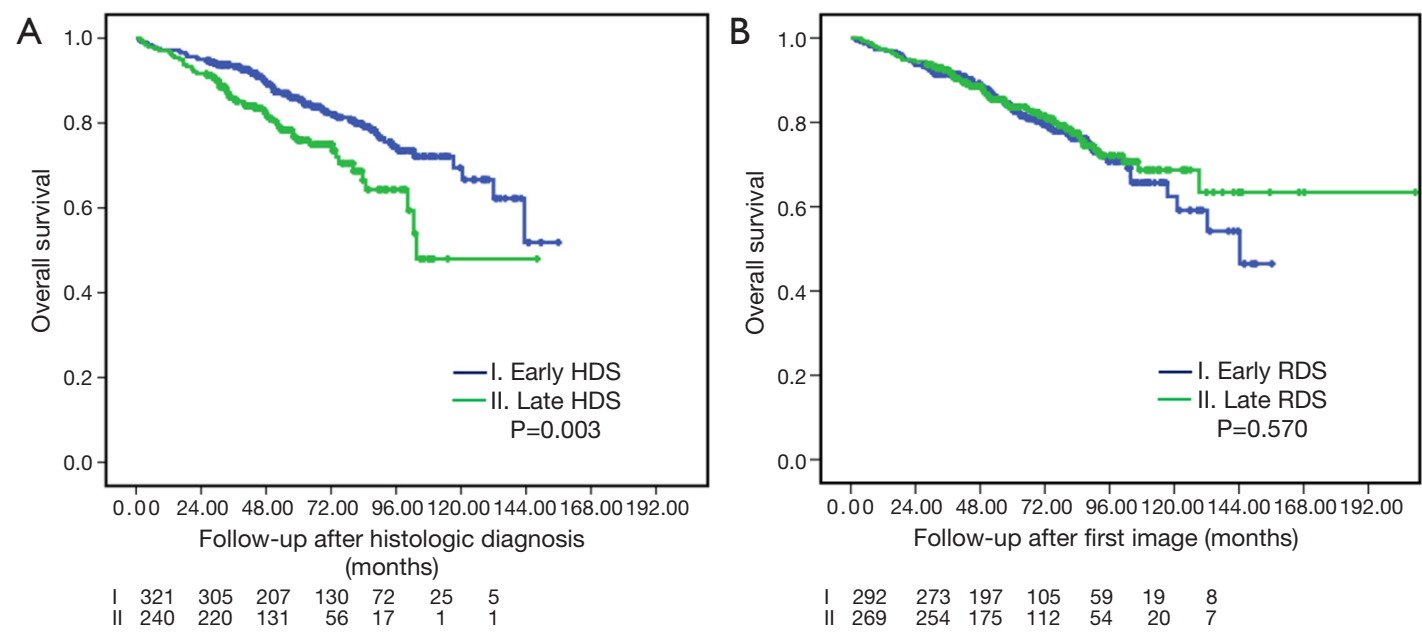

Figure 1 Overall survivals between early and late intervention after histologic (A) and radiologic (B) diagnosis.

an important clinical, social, and political issue. Currently, no guidelines are provided for meaningful clinical delay of surgical resection in early-stage lung cancer. Despite emphasis in the existing cancer care guidelines on the need to avoid delays, no recommendations are given for the optimal initiation time for surgical intervention $(20,21)$. The controversy about outcomes associated with treatment delays in lung cancer may possibly be because of the heterogeneous population, even in stage I NSCLC. Some previous studies have included slow-growing tumors such as adenocarcinoma in situ (AIS) or minimally invasive adenocarcinoma (MIA). In the present study, 55 patients were diagnosed as AIS/MIA, tumors that present as radiologic GGOs and indolent lung cancers with a median time of 22 days (4-93 days) from histologic diagnosis to surgery and 90 days (18-2,611 days) from first image to surgery. Except for one patient in the late-intervention group who died of breast cancer, no other tumor recurrence was found at subgroup analysis between the early and late intervention groups; consequently, no therapeutic delay issue was noted in this subgrouping of early-stage lung cancer. Given that the AIS/MIA tumors present as pure GGO, the prognosis for patients with subsolid and solid nodules is different, as are the invasiveness and growth rate of the tumors. This may be an important parameter to take into account when considering patient outcomes associated with delays in surgical treatment. We further divided the radiologic appearance of the tumors into pure GGO $(0.2<$ CTR $)$, subsolid $(0.2 \leq$ CTR $<0.5)$ and solid $(0.5 \leq$ CTR $\leq 1.0)$. The associations between HDS and RDS treatment delays and OS were also analyzed further. We found that only solid-dominant tumors showed significant differences in OS between early vs. late HDS $(\mathrm{P}=0.003)$ (Figure 3).

Controversy about the timeliness of surgery is directly related to methods used for diagnosis confirmation and the intervals from diagnosis to surgery. In the present study, we compared different OS times associated with surgical outcome time-lags, calculated from the dates of both histologic and radiologic diagnoses. Interestingly, only the time-lag after tissue diagnosis, but not the delayed imaging time-lag, showed significant differences in OS between early and late surgical intervention. The shorter delays in patients with associated favorable outcomes were consistent with those reported in other studies $(9,14,22)$, taking into cognizance the delay from the histologic diagnosis. In contrast, as also shown in previous study results $(8,12)$, results of the present study showed that treatment delays were not associated with worse survival when the delay from the first radiologic study (image-to-surgery) is taken into consideration. To the best of our knowledge, no previous study has reported that delayed RDS has a significant effect on patient outcomes. Accordingly, to evaluate the quality of measures of lung cancer management and treatment for those diagnosed as early-stage lung cancer and considered clinically to be surgical candidates, the date of tissue diagnosis may be a more appropriate "diagnosis date" than the date of image diagnosis when focusing on timely treatment, surgical or medical.

Few previous studies have examined the reasons for delays in lung cancer care $(3,5)$. In the present study, the independent predictors of late HDS included higher comorbidity score, receiving brain MRI screening, and the 
Table 3 Demographics of patients with clinical stage I adenocarcinoma who underwent either early or delayed pulmonary resection (after PSM)

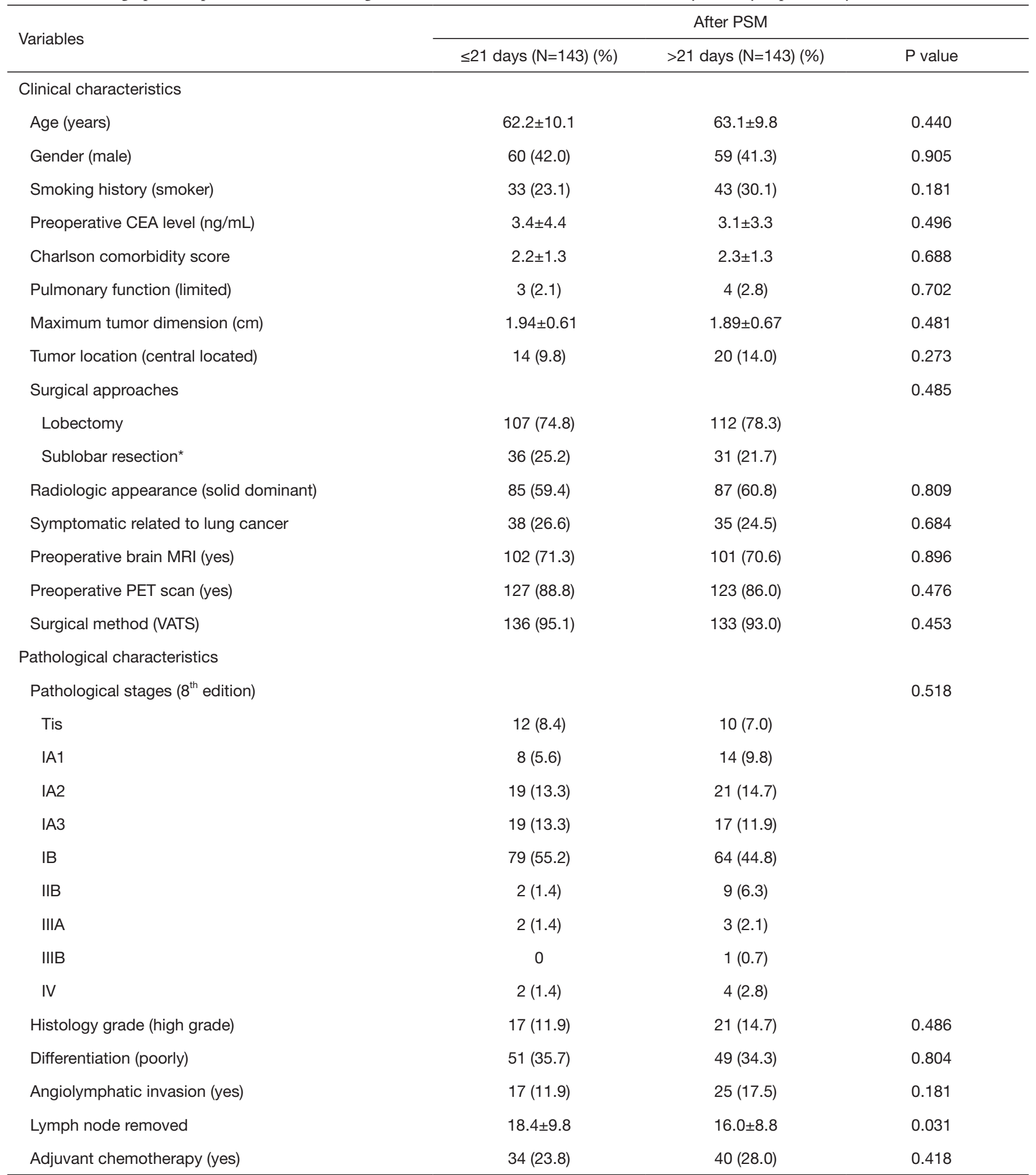

PSM, propensity score matching; CEA, carcinoembryonic antigen; MRI, magnetic resonance imaging; VATS, video-assisted thoracoscopic surgery; *Sublobar resection, wedge + segmentectomy. 
Table 4 Risk analysis of overall survival in 286 clinical stage I adenocarcinoma patients (after PSM)

\begin{tabular}{|c|c|c|c|c|c|c|}
\hline Variables & \multicolumn{3}{|c|}{ Univariate } & \multicolumn{3}{|c|}{ Multivariate } \\
\hline Delayed surgery (>14 days) & 3.660 & $1.310-10.227$ & 0.013 & 2.697 & $0.947-7.684$ & $0.063^{*}$ \\
\hline Delayed surgery (>21 days) & 2.276 & $1.209-4.283$ & 0.011 & 2.031 & $1.041-3.963$ & 0.038 \\
\hline Delayed surgery (>28 days) & 1.931 & $1.074-3.473$ & 0.028 & 1.845 & $1.005-3.387$ & $0.048^{*}$ \\
\hline \multicolumn{7}{|l|}{ Clinical characteristics } \\
\hline Charlson comorbidity score & 1.484 & $1.231-1.790$ & $<0.001$ & 1.610 & $1.068-2.425$ & 0.023 \\
\hline Maximum tumor dimension (cm) & 2.539 & $1.520-4.239$ & $<0.001$ & 2.164 & $1.073-4.365$ & 0.031 \\
\hline Preoperative brain MRI screening (without) & 2.063 & $1.130-3.768$ & 0.018 & 2.051 & $1.016-4.138$ & 0.045 \\
\hline \multicolumn{7}{|l|}{ Pathological characteristics } \\
\hline
\end{tabular}

${ }^{1}$ Calculated by Cox regression method; only variables with $\mathrm{P} \leq 0.1$ after the univariate analyses were entered into the multivariate model. *, adjusted with comorbidity score, maximum tumor dimension, preoperative brain MRI screening and angiolymphatic invasion.
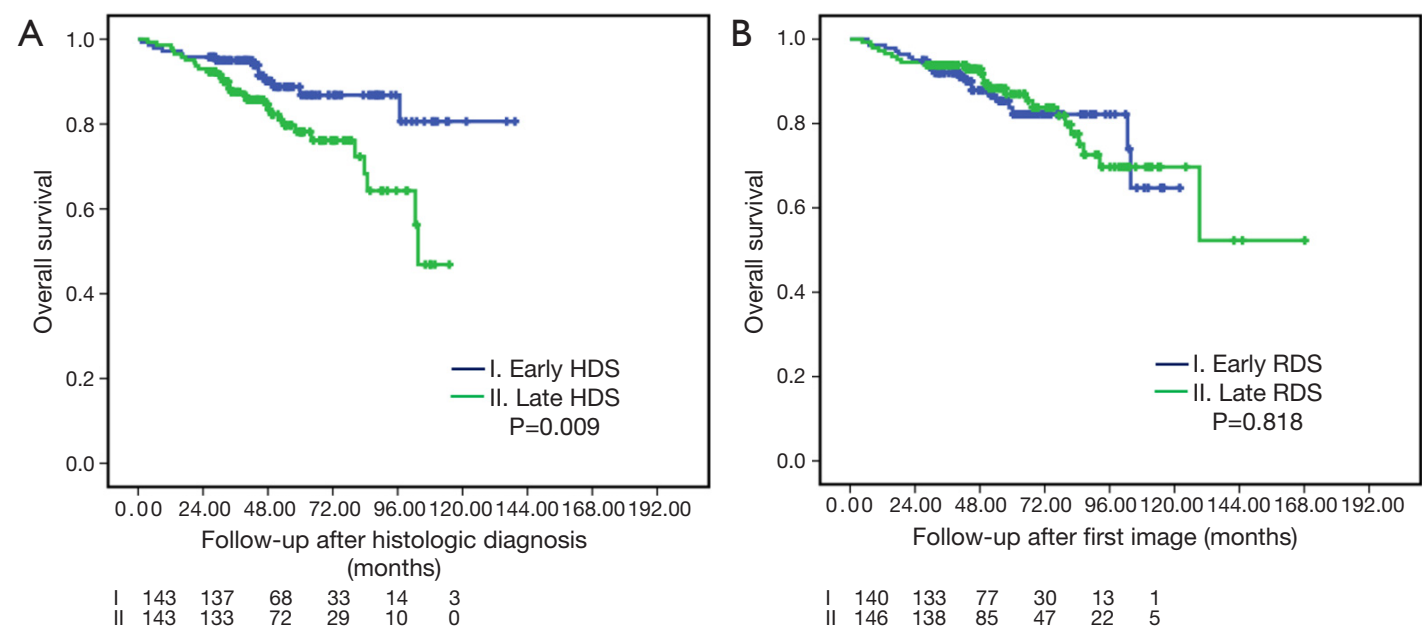

Figure 2 Overall survivals after propensity-score matching. Early and late intervention after histologic (A) and radiologic (B) diagnosis.

VATS approach. It is understandable that patients with a high comorbidity score may take longer to undergo a full work-up for surgery (23), and patients undergoing brain MRI screening may require waiting for time on the scanner. In the present study, more patients underwent pulmonary resection using the VATS approach $(86 \%)$, and waited longer for their surgery compared to those receiving the open method. This may be related to the higher percentage of patients receiving complete staging (including brain MRI and $\mathrm{PET}$ scans, $60 \%$ vs. $15 \%$, respectively; $\mathrm{P}<0.001$ ) after histologic diagnosis according to the individual surgeon's preference.

Furthermore, delayed surgery caused by invasive mediastinal lymph node evaluation, such as mediastinoscopy and EBUS lymph node aspiration were less important in the present study than we had originally hypothesized. This might be attributed to the fact that the majority of our patients $(84 \%)$ had preoperative whole-body PET/CT scans to exclude the possibility of mediastinal lymph node involvement in clinical stage I adenocarcinoma. Although 18 patients underwent mediastinoscopy, the procedure was performed on the same day as that of lung resection; 

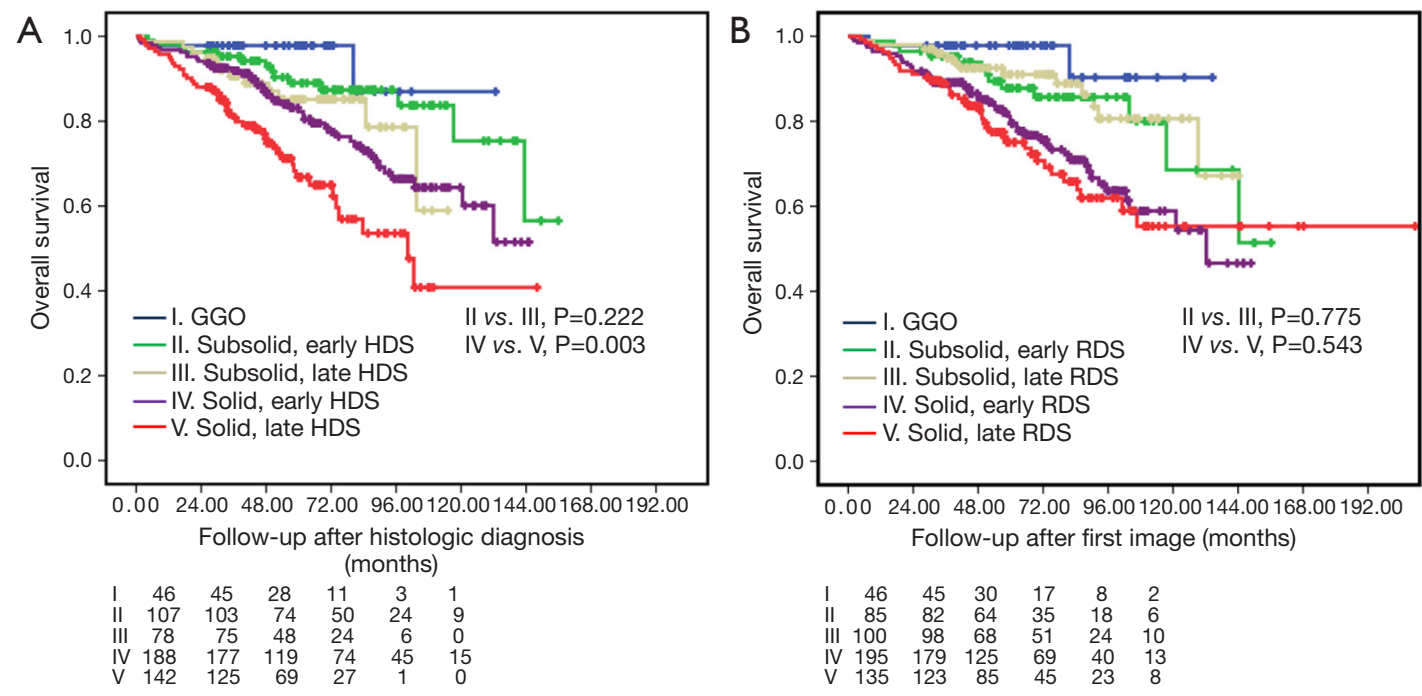

Figure 3 Subgrouping by preoperative radiologic pattern in overall survivals between early and late intervention after histologic (A) and radiologic (B) diagnosis.

only 8 patients received preoperative EBUS lymph node aspiration ( 2 and 6 patients in the early and late HDS group, separately).

In the present study, the cutoff used for HDS (21 days) and RDS (60 days) were both shorter than those reported in other studies $(3,7,9,13,14,22)$. However, the definition "delayed" surgery according to the median HDS time in the entire cohort still aligns with the 8 weeks recommended by the British Thoracic Society (24). However, this exceeds the 14-day target established by the Danish Lung Cancer Registry (25) and is longer than that for studies from Finland (median 15 days) (16), India (median 20 days) (26), and Turkey (median 12 days) (27). Obviously, different countries or areas have different facilities and medical systems to manage their lung cancer patients; thus, regional variations exist in the timelines of lung cancer care.

The main strength of the present study is restricting the focus to preoperative histologic-diagnosed clinical stage I adenocarcinoma only, rather than ambiguous definitions regarding lung cancer "diagnosis" $(5,13,14,22)$ from singlecenter institutes. By using precise diagnostic criteria, Kasymjanova et al. (9) reported significant differences in median survival in early stage NSCLC between the 30 day "diagnosis-to-treatment" intervals. The present study also restricted patients to adenocarcinoma only, which has grown to be the leading histopathologic type of lung cancer currently. Therefore, the present study results could be directly applied to this specific patient population. Of note, no previous studies specifically evaluated the impact of delayed surgical treatment after histologic and radiologic diagnoses on lung cancer patients with clinical stage I adenocarcinoma.

Maiga et al. (3) reported no associations between tumor growth and time to treatment among 265 NSCLC patients (197 preoperative diagnoses) who underwent surgical resection. Although not significant, a higher tumor stage progression rate $(30 \%$ vs. $22 \%)$ was noted with delayed surgery of $>60$ days compared to earlier surgery (3). Similar observations were also made in the present study between later and earlier surgeries $(10.4 \%$ vs. $7.4 \%, \mathrm{P}=0.222)$. In contrast, Samson et al. (11) concluded that delays in resection are independently associated with increased rates of upstaging and decreased median survival. Briefly, except for tumor growth and stage migration that occur following delays to treatment, another mechanism involving associations between worse OS and late HDS, but not late RDS, may be the destructive basement membrane of tumors after histologic confirmation, which may result in occult metastasis through lymphatic or hematogenous dissemination (28).

\section{Limitations}

The major limitation of this study was its retrospective nature based on analysis of data from a single institute. With confirmation of the study findings through additional studies, renewed emphasis on expedited 
timely resection may support meaningful improvement in oncologic outcomes. The results of the present study may not be applicable to symptomatic or advanced lung cancer, especially with other histologic findings. Finally, information regarding healthcare costs, regional geographic differences, and socioeconomic evaluation were lacking and were therefore not evaluated; however, the majority of medical costs are covered by the National Health Insurance that covers $99 \%$ of the population.

\section{Conclusions}

In clinical stage I lung adenocarcinoma, late HDS but not late RDS was an independent predictor of worse OS. Our results may help to improve the level of evidence available to support further treatment guidelines and management of stage I lung adenocarcinoma. The need for streamlined patient referral, or development of a multidisciplinary, centralized referral program may be necessary to minimize delayed surgery among early stage lung cancer patients who are surgical candidates. Additionally, intense followup, instead of histologic diagnosis, may reduce the time of treatment delays for patients who wish to delay or are uncertain about proceeding with surgical intervention. Future studies are needed to determine the optimal timing of treatment for different stages of NSCLC as well as for cases with other histology.

\section{Acknowledgments}

Funding: None.

\section{Footnote}

Conflicts of Interest: The authors have no conflicts of interest to declare.

Ethical Statement: The authors are accountable for all aspects of the work in ensuring that questions related to the accuracy or integrity of any part of the work are appropriately investigated and resolved. The study protocol was approved by the hospital Institutional Review Board and the informed consent requirement was waived (approval no. 2019-04-009AC).

Open Access Statement: This is an Open Access article distributed in accordance with the Creative Commons Attribution-NonCommercial-NoDerivs 4.0 International
License (CC BY-NC-ND 4.0), which permits the noncommercial replication and distribution of the article with the strict proviso that no changes or edits are made and the original work is properly cited (including links to both the formal publication through the relevant DOI and the license). See: https://creativecommons.org/licenses/by-nc-nd/4.0/.

\section{References}

1. Kaplan GS. Health care scheduling and access: A report from the IOM. JAMA 2015;314:1449-50.

2. American Cancer Society. Facts \& Figures 2019: American Cancer Society. Available online: https://www.cancer. org/content/dam/cancer-org/research/cancer-facts-andstatistics/annual-cancer-facts-and-figures/2019/cancerfacts-and-figures-2019.pdf. Accessed January 8, 2019.

3. Maiga AW, Deppen SA, Pinkerman R, et al. Timeliness of care and lung cancer tumor-stage progression: How long can we wait? Ann Thorac Surg 2017;104:1791-7.

4. Devbhandari MP, Quennell P, Krysiak P, et al. Implications of a negative bronchoscopy on waiting times to treatment for lung cancer patients: results of a prospective tracking study. Eur J Cardiothorac Surg 2008;34:479-83.

5. Gomez DR, Liao KP, Swisher SG, et al. Time to treatment as a quality metric in lung cancer: Staging studies, time to treatment, and patient survival. Radiother Oncol 2015;115:257-63.

6. Schoenmaekers JJ, Dingemans AC, Hendriks LEL. Brain imaging in early stage non-small cell lung cancer: Still a controversial topic? J Thorac Dis 2018;10:S2168-71.

7. Aragoneses FG, Moreno N, Leon P, et al. Influence of delays on survival in the surgical treatment of bronchogenic carcinoma. Lung Cancer 2002;36:59-63.

8. Quarterman RL, McMillan A, Ratcliffe MB, et al. Effect of preoperative delay on prognosis for patients with early stage non-small cell lung cancer. J Thorac Cardiovasc Surg 2003;125:108-13.

9. Kasymjanova G, Small D, Cohen V, et al. Lung cancer care trajectory at a canadian centre: An evaluation of how wait times affect clinical outcomes. Curr Oncol 2017;24:302-9.

10. Coughlin S, Plourde M, Guidolin K, et al. Is it safe to wait? The effect of surgical wait time on survival in patients with non-small cell lung cancer. Can J Surg 2015;58:414-8.

11. Samson P, Patel A, Garrett T, et al. Effects of delayed surgical resection on short-term and long-term outcomes in clinical stage I non-small cell lung cancer. Ann Thorac Surg 2015;99:1906-12. 
12. Diaconescu R, Lafond C, Whittom R. Treatment delays in non-small cell lung cancer and their prognostic implications. J Thorac Oncol 2011;6:1254-9.

13. Vinod SK, Chandra A, Berthelsen A, et al. Does timeliness of care in non-small cell lung cancer impact on survival? Lung Cancer 2017;112:16-24.

14. Yang CJ, Wang H, Kumar A, et al. Impact of timing of lobectomy on survival for clinical stage Ia lung squamous cell carcinoma. Chest 2017;152:1239-50.

15. Kanarek NF, Hooker CM, Mathieu L, et al. Survival after community diagnosis of early-stage non-small cell lung cancer. Am J Med 2014;127:443-9.

16. Salomaa ER, Sallinen S, Hiekkanen H, et al. Delays in the diagnosis and treatment of lung cancer. Chest 2005;128:2282-8.

17. Bryant AS, Cerfolio RJ. Differences in outcomes between younger and older patients with non-small cell lung cancer. Ann Thorac Surg 2008;85:1735-9.

18. Ha D, Ries AL, Montgrain P, et al. Time to treatment and survival in veterans with lung cancer eligible for curative intent therapy. Respir Med 2018;141:172-9.

19. Hattori A, Matsunaga T, Takamochi K, et al. Neither maximum tumor size nor solid component size is prognostic in part-solid lung cancer: Impact of tumor size should be applied exclusively to solid lung cancer. Ann Thorac Surg 2016;102:407-15.

20. Vansteenkiste J, Crino L, Dooms C, et al. 2nd ESMO consensus conference on lung cancer: Early-stage nonsmall-cell lung cancer consensus on diagnosis, treatment and follow-up. Ann Oncol 2014;25:1462-74.

Cite this article as: Huang CS, Hsu PK, Chen CK, Yeh YC, Shih CC, Huang BS. Delayed surgery after histologic or radiologic-diagnosed clinical stage I lung adenocarcinoma. J Thorac Dis 2020;12(3):615-625. doi: 10.21037/jtd.2019.12.123
21. Howington JA, Blum MG, Chang AC, et al. Treatment of stage I and II non-small cell lung cancer: Diagnosis and management of lung cancer, 3rd ed. American College of Chest Physicians evidence-based clinical practice guidelines. Chest 2013;143:e278S-e313S.

22. Bott MJ, Patel AP, Crabtree TD, et al. Pathologic upstaging in patients undergoing resection for stage I nonsmall cell lung cancer: Are there modifiable predictors? Ann Thorac Surg 2015;100:2048-53.

23. Damhuis RA, Maat AP, Plaisier PW. Performance indicators for lung cancer in the Netherlands. Eur J Cardiothorac Surg 2015;47:897-903.

24. BTS recommendations to respiratory physicians for organising the care of patients with lung cancer. The Lung Cancer Working Party of the British Thoracic Society Standards of Care Committee. Thorax 1998;53:S1-8.

25. Jakobsen E, Green A, Oesterlind K, et al. Nationwide quality improvement in lung cancer care: The role of the Danish lung cancer group and registry. J Thorac Oncol 2013;8:1238-47.

26. Chandra S, Mohan A, Guleria R, et al. Delays during the diagnostic evaluation and treatment of lung cancer. Asian Pac J Cancer Prev 2009;10:453-6.

27. Annakkaya AN, Arbak P, Balbay O, et al. Effect of symptom-to-treatment interval on prognosis in lung cancer. Tumori 2007;93:61-7.

28. Nakajima J. Preoperative pathological diagnosis of lung cancer: Is it always necessary? Ann Thorac Cardiovasc Surg 2012;18:183-5. 


\section{Supplementary}

Table S1 Independent predictors of late RDS in 561 clinical stage I adenocarcinoma

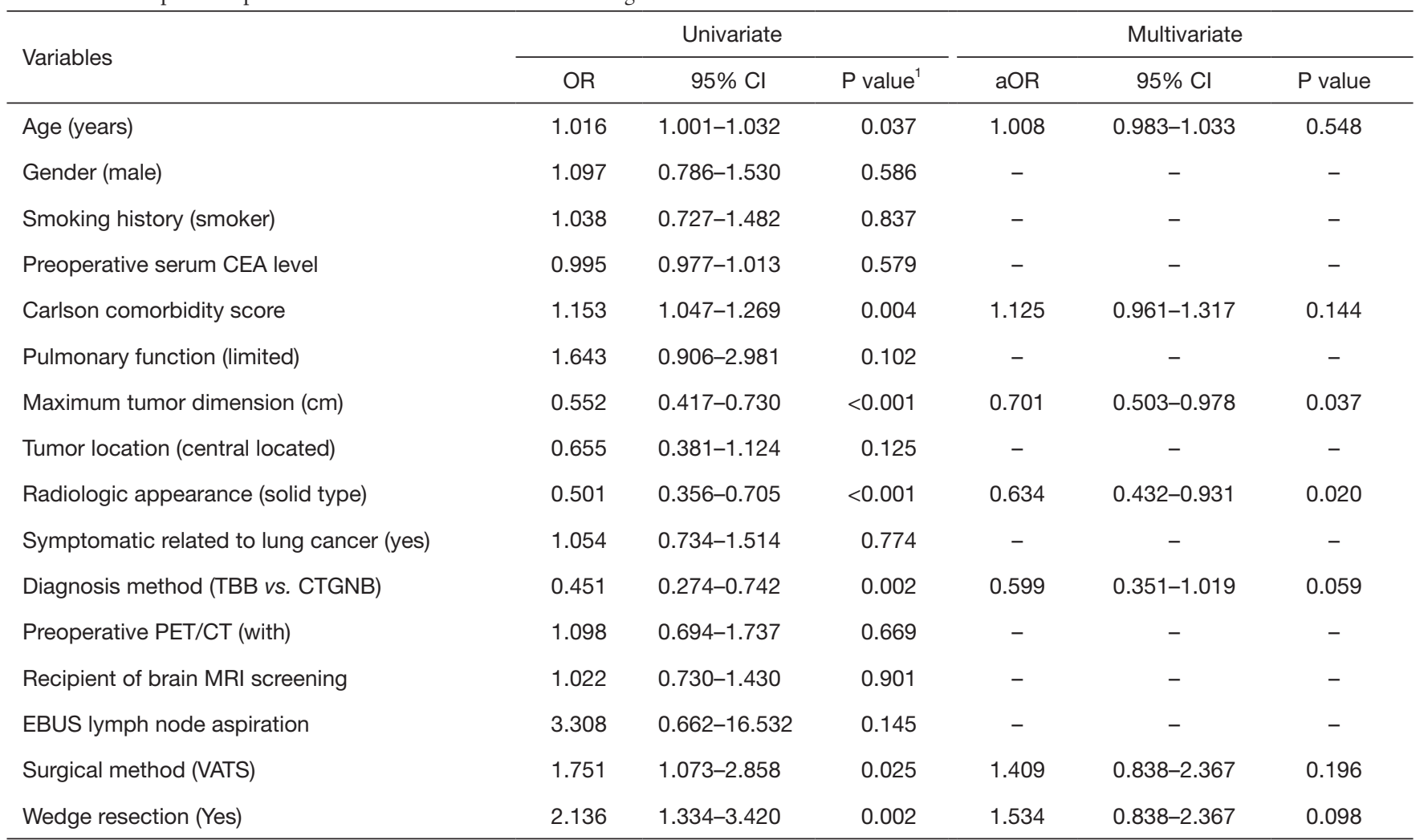

${ }^{1}$ Calculated by Logistic regression method; only variables with $\mathrm{P} \leq 0.1$ after the univariate analyses were entered into the multivariate model. OR, odds ratio; Cl, confidence interval; aOR, adjusted odds ratio; CEA, carcinoembryonic antigen; TBB, transbronchial biopsy; CTGNB, computed tomography guided needle biopsy; PET, positron emission tomography; MRI, magnetic resonance imaging; EBUS, endobronchial ultrasound; VATS, video-assisted thoracoscopic surgery. 